\title{
Computer Applications of Brahmagupta-Bhãskara Equation
}

\author{
Tarun Kumar Gupta \\ Department of Mathematics and Statistics \\ Gurukula Kangri University, Haridwar (U.K), India
}

\begin{abstract}
In this paper, we propose a new interpretation of Brahmagupta's terminology for Computer programming of different values of $\mathrm{N}$ and $\mathrm{m}$. we also extend some Vedic composition tables (for the values of $\mathrm{g}_{\mathrm{N}}(\mathrm{m})$ ) with Maple code that hold for the "Bhāvanā".
\end{abstract}

\section{Keywords}

Brahmagupta's Bhãskara equation, Maple Software, Bhāvanā, $\mathrm{g}_{\mathrm{N}}(\mathrm{m})$.

*Corresponding Author

\section{INTRODUCTION}

The Brahmagupta-Bhãskara (BB) equation is a Second order indeterminate equation of the forms

(i) $\mathrm{Nx}^{2}+\mathrm{k}=\mathrm{y}^{2}$ (Verga prakrti ) (ii) $\mathrm{Ny}^{2}+\mathrm{k}=\mathrm{x}^{2}$ (iii) $\mathrm{Ny}^{2}+1=\mathrm{x}^{2}$ the word "prakrti" means coefficient and refers to the coefficient $\mathrm{N}$ is this equation.

Where $\mathrm{k}$ is an integer and $\mathrm{N}$ is a positive integer and not a perfect square [1]. A particular case of the above BB-equation with $\mathrm{k}=1$ is known as Pell equations. Indeed the equation $\mathrm{Ny}^{2}+\mathrm{k}=\mathrm{x}^{2}$ even now bears incorrectly the name of John Pell (AD 1610-1685), an English mathematician, although connection with it consists of simply the publications of the solutions of it in his edition of Brouncker's translation of Rohinus's Algebra (AD 1668) [6]. It was an accident that Leonhard Euler (AD 1707-1783), the famous Swiss mathematician, referred to this as the Pell equation even now has no historical justification. To continue to call this equation "Pell equation" is a misnomer. It is fitting and justified that this "Varga- prakrti" equation should be renamed as Brahmagupta- Bhãskara equation [7].

Michael Atiyah says that "Number theory for its own sake, as a great intellectual challenge, has a long history, particularly here in India. Already in the $7^{\text {th }}$ centuary, Brahmagupta made important contributions to what is now known (incorrectly) as Pell's equation [11].

\section{DEFINE $\mathrm{g}_{\mathbf{N}}(\mathrm{m})$ WITH $B H \bar{A} V A N \bar{A}$}

The reduction modulo $\mathrm{m}$ map $\operatorname{red}_{m}: \mathbb{Z} \rightarrow \mathbb{Z} / m \mathbb{Z}$ where $\mathrm{m}>1$ belongs to $\mathrm{I}$ is the map that sends an integer a to its remainder $r(0 \leq r \leq m)$ on division by $m$. We shall denote the image of $\operatorname{red}_{\mathrm{m}}$ by putting bar on the element of $\mathbb{Z}$. For $m=5$, then $\operatorname{red}_{5}(16)=\overline{1}$ and $\operatorname{red}_{5}(-22)=\overline{3}$. The $\operatorname{map} \operatorname{red}_{m}$ is a ring homomorphism. Furthermore, if $\mathrm{p}$ is prime, then the set of remainders upon division by $\mathrm{p}, \mathbb{Z} / \mathrm{pZ}$ is a field. All that we need to check is that every element has an inverse. To see that every element in $\mathbb{Z} / \mathrm{p} \mathbb{Z}$ has an inverse, notice that is $0<\mathrm{a}<\mathrm{p}$,

\author{
Nidhi Handa \\ Department of Mathematics and Statistics \\ Gurukula Kangri University, Haridwar (U.K), India
}

then $\operatorname{gcd}(\mathrm{a}, \mathrm{p})=1$. By the Euclidean algorithm, we can write $\mathrm{px}+\mathrm{ay}=1$ for some integers $\mathrm{x}$ and $\mathrm{y}$. Upon reduction modulo $\mathrm{p}$ of this equation, we see that $\bar{a} \bar{y}=1$ and $\bar{y} \in \mathbb{Z} / p \mathbb{Z}$. The map $\operatorname{red}_{\mathrm{m}}$ induces a ring homomorphism on the ring of matrices also denoted by [4].

$$
\operatorname{red}_{m}: \mathrm{M}_{2}(\mathbb{Z}) \rightarrow \mathrm{M}_{2}(\mathbb{Z} / \mathrm{mZ})
$$

Where $\mathrm{M}_{2}(\mathbb{Z})$ and $\mathrm{M}_{2}(\mathbb{Z} / \mathrm{mZ})$ are the rings of $2 \times 2$ matrices with entries in $\mathbb{Z}$ and $\mathbb{Z} / \mathrm{mZ}$, respectively. For example, it is not difficult to see that.

$$
\operatorname{red}_{5}\left(\begin{array}{cc}
6 & 5 \\
23 & -2
\end{array}\right)=\left(\begin{array}{cc}
\overline{1} & \overline{0} \\
\overline{3} & \overline{3}
\end{array}\right)
$$

Similarly, given the map $\mathrm{G} \rightarrow \mathrm{SL}_{2}(\mathbb{Z}), \operatorname{red}_{\mathrm{m}}$ also induces a group homomorphism [5].

$$
\operatorname{red}_{m}: \mathrm{G} \rightarrow \mathrm{SL}_{2}(\mathbb{Z} / \mathrm{mZ})
$$

The image of this map is clearly a finite group. Moreover, since $\mathrm{G}$ is cyclic, $\mathrm{red}_{\mathrm{m}}$ is a finite cyclic group. We denote by $\mathrm{g}_{\mathrm{N}}(\mathrm{m})$ the order of the image of $\mathrm{G}$ under the reduction mod $\mathrm{m}$ homomorphism. In the following discussion, since each element in the image $\operatorname{red}_{m}(G)$ is of the form [5].

$$
\left(\begin{array}{cc}
\bar{x} & \bar{y} \\
\bar{N} \bar{y} & \bar{x}
\end{array}\right)
$$

We will denote these elements simply by $(\mathrm{x}, \mathrm{y})$. The group law defining $\mathrm{G}$ as a group is equivalent to the corresponding matrix multiplication, so when we multiply elements of $\operatorname{red}_{\mathrm{m}}$ (G) we will simply use [4].

\subsection{Brahmagupta's Bhāvanā (The Principal of Composition)}

The solution space of the equation $\mathrm{x}^{2}-\mathrm{Ny}^{2}=1$ admits the Binary operations [2].

$\left(\mathrm{x}_{1}, \mathrm{y}_{1}\right) \odot\left(\mathrm{x}_{2}, \mathrm{y}_{2}\right)=\left(\mathrm{x}_{1} \mathrm{x}_{2}+\mathrm{Ny}_{1} \mathrm{y}_{2}, \mathrm{x}_{1} \mathrm{y}_{2}+\mathrm{x}_{2} \mathrm{y}_{1}\right)$

Here we will give an example of how $\mathrm{g}_{\mathrm{N}}(\mathrm{m})$ can be computed with Vedic composition table.

Let us consider $\mathrm{x}^{2}-\mathrm{Ny}^{2}=1 \quad$ (for $\mathrm{N}=7$ ) the first integer solution to this equation and therefore a generator of $G$, is $(8,3)$. Now we want to consider $\operatorname{red}_{5}(\mathrm{G})$. We will use bar notation to indicate elements of $\mathbb{Z} / 5 \mathbb{Z}$. Upon reduction, the generator becomes $(\overline{3}, \overline{3})$. Now we know that red5 (G) is cyclic, so to find the order of the group. It suffices to multiply the generator until we get back to the identity, $(\overline{1}, \overline{0})$ 
Vedic Composition Table: 2.1

\begin{tabular}{|l|l|l|l|}
\hline S.NO & $\begin{array}{l}\text { Modulo 5 } \\
\text { N=7 }\end{array}$ & $(\overline{3}, \overline{3})$ & $\begin{array}{l}\text { Third Vedic Sutra } \\
\text { Vertically and } \\
\text { Crosswise } \\
\text { **'Bhāvanā" }\end{array}$ \\
\hline 1. & $(\overline{3}, \overline{3})$ & $\begin{array}{l}(\overline{72}, \overline{18}) \\
(\overline{2}, \overline{3})\end{array}$ & $\begin{array}{l}(\overline{69}, \overline{15}) \\
(\overline{4}, \overline{0})\end{array}$ \\
\hline 2. & $(\overline{3}, \overline{3})$ & $\begin{array}{l}(\overline{12}, \overline{12}) \\
(\overline{2}, \overline{2})\end{array}$ \\
\hline 3. & $(\overline{3}, \overline{3})$ & $(\overline{48}, \overline{12})$ & $\vdots$ \\
$(\overline{3}, \overline{2})$ & $\vdots$ \\
\hline 5. & $(\overline{3}, \overline{3})$ & $(\overline{51}, \overline{15})$ & $\vdots$ \\
\hline
\end{tabular}

So we see that the order of $\operatorname{red}_{5}(G)$ is 6 , that is $g_{7}(5)=6$.

**It is similar to third Vedic Sutra that is "Urdhavtriyakbhyam" means Vertically and Crosswise from 16-Vedic Sutra [8].

\section{THE COMPUTATION OF $\mathrm{g}_{\mathrm{N}}(\mathrm{m})$ WITH MAPLE PROCEDURES}

Calculating $\mathrm{g}_{\mathrm{N}}(\mathrm{m})$ can also require a great deal work. It is best done using a computer, especially for large values of $\mathrm{N}$ and $\mathrm{m}$.

The following procedures use Brahamgupta's Bhāvanā to produce as many tables to a particular BB-equation (Pell-Like equation) as desired. Following are list of commands, which can be useful for computing solutions of BB-equation, and $\mathrm{g}_{\mathrm{N}}(\mathrm{m})$.

To begin we need the following packages [10] restart; with (numtheory) [9]

The command NGen takes an integer (preferably square free) and returns the generator of $\mathrm{G}$ as a list.

NGen :=proc (N: : integer)

$\mathrm{h}$ :mMult $(\mathrm{g},[1,0], \mathrm{N}, \mathrm{m})$;

while not $\mathrm{h}=[1,0]$ do

group : = [op (group), $\mathrm{h}]$;

$\mathrm{h}:=$ mMult $(\mathrm{h}, \mathrm{g}, \mathrm{N}, \mathrm{m})$

end do;

group : = [op (group), $\mathrm{h}]$

return [group , nops (group) , N, m ]

end proc :

The command GN accepts the same input as the previous command. It returns the order of the $\operatorname{group} \operatorname{red}_{\mathrm{m}}(\mathrm{G})$.

$\mathrm{GN}:=\operatorname{proc}(\mathrm{N}::$ integer $, \mathrm{m}::$ integer $)$

local $\mathrm{g}, \mathrm{k}, \mathrm{h}$;

$\mathrm{g}:=\mathrm{NGen}(\mathrm{N})$;

$\mathrm{h}:=\operatorname{mMult}(\mathrm{g},[1,0], \mathrm{N}, \mathrm{m})$; local cf, z, x,y, j,test,i ;

$\mathrm{cf}:=\operatorname{cfrac}(\operatorname{sqrt}(\mathrm{N}))$;

$\mathrm{x}:=$ nthnumer $(\mathrm{cf}, 1)$;

$\mathrm{y}:=$ nthdenom $(\mathrm{cf}, 1)$;

test $:=$ false $; \mathrm{i}:=1$;

while test $=$ false do

if $x^{\wedge} 2-\mathrm{Ny}^{\wedge} 2=1$

then test : $=$ true

else i : $=\mathrm{i}+1$;

cf : $=\operatorname{cfrac}(\operatorname{sqrt}(\mathrm{N}), \mathrm{i})$;

$\mathrm{x}:=$ nthnumer $(\mathrm{cf}, 1)$;

$\mathrm{y}:=$ nthdenom $(\mathrm{cf}, 1)$;

end if

end do ;

return $[\mathrm{x}, \mathrm{y}]$

end proc :

The command NMult accepts as input two lists (these should be solutions to (1)) and an integer $(\mathrm{N})$. It multipies the two lists according to the principal of composition "Bhâvana" defined for $\mathrm{G}$, and returns the product as a list. The command mMult accepts as input two lists ( these should be solutions to (1) and an integer $(\mathrm{N})$ and an integer $(\mathrm{m})$. It performs the multiplication $\bmod \mathrm{m}$, and then returns the product as a list [7].

NMult : = proc $(\mathrm{x} 1::$ list, $\mathrm{x} 2::$ list, $\mathrm{N})$

return $\left[\mathrm{x} 1[1] * \mathrm{x} 2[1]+\mathrm{N}^{*} \mathrm{x} 1[2] * \mathrm{x} 2[2], \mathrm{x} 1[1] * \mathrm{x} 2[2]+\right.$ $\mathrm{x} 1[2] * \mathrm{x} 2[1]]$

end proc :

mMult : = proc $(\mathrm{x} 1::$ list $, \mathrm{x} 2::$ list $, \mathrm{N}::$ integer $, \mathrm{m}::$ integer $)$ return $\left[\bmod \left(\mathrm{x} 1[1] * \mathrm{x} 2[1]+\mathrm{N}^{*} \mathrm{x} 1[2] * \mathrm{x} 2[2], \mathrm{m}\right), \bmod (\mathrm{x} 1[1]\right.$ $* x 2[2]+x 1[2] * x 2[1]], m)]$

end proc :

The command mGroup accepts as input two integers. The first is $\mathrm{N}$, and the second is the integer $\mathrm{m}$, which will be used in reduction $\bmod \mathrm{m}$. it returns a list of group elements (each presented as a list with two elements), $\mathrm{g}_{\mathrm{N}}(\mathrm{m}), \mathrm{N}$ and $\mathrm{m}$.

mGroup: $=$ proc $(\mathrm{N}::$ integer $, \mathrm{m}::$ integer $)$

local $\mathrm{g}$, group, $\mathrm{h}$;

group : $=[]$;

$\mathrm{g}:=\mathrm{NGen}(\mathrm{N})$;

$\mathrm{k}:=1$; while $\operatorname{not} \mathrm{h}=[1,0]$ do

$\mathrm{h}:=\operatorname{mMult}(\mathrm{h}, \mathrm{g}, \mathrm{N}, \mathrm{m})$

$\mathrm{k}:=\mathrm{k}+1$

end do;

return $\mathrm{k}$

end proc :

The command mGroup can take some time to run, because of the inherent difficulty in finding the generator for the group $\mathrm{G}$. If the generator is known, then mGroupGen will accept two integers $(\mathrm{N}$ and $\mathrm{m})$ and a generator as input, and build the group generated by this element. It performs a check that the alleged generator is in fact a solution of (1). This command can save on time if the generator for $\mathrm{G}$ is difficult to compute. It returns the same as the previous command [3]. 
mGroupGen $:=\operatorname{proc}(\mathrm{N}::$ integer $, \mathrm{m}::$ integer, gen $::$ list $)$ local group, $\mathrm{h}$;

group : $=[$;

if gen $[1]^{\wedge} 2-\mathrm{N}^{*}$ gen $[2]^{\wedge} 2=1$

then $\mathrm{h}:=$ mMult $($ gen $,[1,0], \mathrm{N}, \mathrm{m})$;

while not $\mathrm{h}=[1,0]$ do

group : $=[$ op(group), $\mathrm{h}]$;

$\mathrm{h}:=$ mMult $(\mathrm{h}$, gen $, \mathrm{N}, \mathrm{m})$

end do;

group : = $[$ op $($ group $), \mathrm{h}]$;

return [group , nops (group) , N, m ]

else print (" the input should be a solution to BB-equation") end if

end proc :

\subsection{Vedic Composition Tables with Maple Procedures (Main results)}

I have included eight tables listing values of $g_{N}(m)$. Table 1 shows $\mathrm{g}_{\mathrm{N}}(\mathrm{p})$ for the first 10 primes. There, I have taken the first square-free integers less than or equal to 30 for N. Table 2 shows $g_{N}\left(p^{k}\right)$ with $1 \leq k \leq 3$ for the first several primes. Again, I have taken the first square free integers less than equal to 30 . Tables 3-8 show the values of $g_{N}(m)$ for the integers $2 \leq m \leq 60$.

Vedic Composition Table: 1 $\mathrm{g}_{\mathrm{N}}(\mathrm{p})$ for the first 10 primes

\begin{tabular}{|c|c|c|c|c|c|c|c|c|c|c|}
\hline $\mathbf{g}_{\mathbf{N}}(\mathbf{p})$ & $\mathbf{p}=2$ & 3 & 5 & 7 & 11 & 13 & 17 & 19 & 23 & 29 \\
\hline $\mathrm{N}=2$ & 1 & 4 & 6 & 3 & 12 & 14 & 8 & 20 & 11 & 10 \\
\hline 3 & 2 & 6 & 3 & 8 & 10 & 12 & 18 & 5 & 11 & 15 \\
\hline 5 & 1 & 4 & 10 & 8 & 5 & 14 & 6 & 3 & 8 & 7 \\
\hline 6 & 1 & 6 & 4 & 8 & 3 & 7 & 18 & 18 & 11 & 28 \\
\hline 7 & 2 & 2 & 6 & 7 & 12 & 14 & 3 & 18 & 12 & 28 \\
\hline 10 & 1 & 1 & 10 & 8 & 12 & 3 & 18 & 4 & 24 & 30 \\
\hline 11 & 2 & 1 & 4 & 3 & 22 & 7 & 18 & 6 & 24 & 15 \\
\hline 13 & 1 & 1 & 2 & 8 & 4 & 26 & 8 & 20 & 11 & 14 \\
\hline 14 & 1 & 4 & 4 & 7 & 10 & 12 & 9 & 20 & 12 & 6 \\
\hline 15 & 2 & 3 & 10 & 6 & 10 & 7 & 16 & 20 & 24 & 30 \\
\hline 17 & 1 & 4 & 6 & 8 & 4 & 6 & 34 & 9 & 24 & 30 \\
\hline 19 & 2 & 2 & 4 & 8 & 3 & 1 & 4 & 38 & 8 & 15 \\
\hline 21 & 1 & 1 & 4 & 14 & 4 & 14 & 16 & 10 & 8 & 5 \\
\hline 22 & 1 & 2 & 3 & 1 & 22 & 12 & 18 & 5 & 24 & 28 \\
\hline 23 & 2 & 4 & 2 & 3 & 10 & 12 & 9 & 18 & 23 & 28 \\
\hline 26 & 1 & 4 & 1 & 8 & 5 & 26 & 4 & 9 & 11 & 30 \\
\hline 29 & 1 & 4 & 1 & 1 & 4 & 2 & 6 & 20 & 21 & 58 \\
\hline 30 & 1 & 6 & 5 & 6 & 4 & 12 & 16 & 9 & 3 & 14 \\
\hline
\end{tabular}

Vedic Composition Table: 2 $g_{N}(p)$ for the first several prime powers

\begin{tabular}{|c|c|c|c|c|c|c|c|c|c|}
\hline $\mathbf{g}_{\mathbf{N}}(\mathbf{p})$ & $\mathbf{m}=2$ & 4 & 8 & 3 & 9 & 27 & 5 & 25 & 125 \\
\hline $\mathbf{N}=2$ & 1 & 2 & 4 & 4 & 12 & 36 & 6 & 30 & 150 \\
\hline 3 & 2 & 4 & 4 & 6 & 18 & 54 & 3 & 15 & 75 \\
\hline 5 & 1 & 1 & 2 & 4 & 4 & 12 & 10 & 50 & 250 \\
\hline 6 & 1 & 2 & 4 & 6 & 6 & 18 & 4 & 20 & 100 \\
\hline 7 & 2 & 4 & 4 & 2 & 6 & 18 & 6 & 30 & 150 \\
\hline 10 & 1 & 2 & 4 & 1 & 3 & 9 & 10 & 50 & 250 \\
\hline 11 & 2 & 4 & 4 & 1 & 3 & 9 & 4 & 20 & 100 \\
\hline 13 & 1 & 1 & 2 & 1 & 1 & 3 & 2 & 10 & 50 \\
\hline 14 & 1 & 2 & 2 & 4 & 12 & 36 & 4 & 20 & 100 \\
\hline 15 & 2 & 4 & 4 & 3 & 3 & 9 & 10 & 50 & 250 \\
\hline 17 & 1 & 1 & 1 & 4 & 12 & 36 & 6 & 30 & 150 \\
\hline 19 & 2 & 4 & 4 & 2 & 6 & 18 & 4 & 20 & 100 \\
\hline 21 & 1 & 2 & 2 & 1 & 3 & 9 & 4 & 20 & 100 \\
\hline 22 & 1 & 2 & 4 & 2 & 6 & 18 & 3 & 15 & 75 \\
\hline 23 & 2 & 4 & 4 & 4 & 12 & 36 & 2 & 10 & 50 \\
\hline 26 & 1 & 2 & 4 & 4 & 12 & 36 & 1 & 5 & 25 \\
\hline 29 & 1 & 1 & 2 & 4 & 4 & 4 & 1 & 5 & 25 \\
\hline 30 & 1 & 2 & 4 & 6 & 18 & 54 & 5 & 25 & 125 \\
\hline
\end{tabular}


Vedic Composition Table: 3

\section{$2 \leq \mathrm{m} \leq \mathbf{1 1}$ and $\mathrm{N} \leq 30$}

\begin{tabular}{|c|c|c|c|c|c|c|c|c|c|c|}
\hline $\mathbf{g}_{\mathbf{N}}(\mathbf{m})$ & $\mathbf{m}=2$ & 3 & 4 & 5 & 6 & 7 & 8 & 9 & 10 & 11 \\
\hline $\mathbf{N}=2$ & 1 & 4 & 2 & 6 & 4 & 3 & 4 & 12 & 6 & 12 \\
\hline 3 & 2 & 6 & 4 & 3 & 6 & 8 & 4 & 18 & 6 & 10 \\
\hline 5 & 1 & 4 & 1 & 10 & 4 & 8 & 2 & 4 & 10 & 5 \\
\hline 6 & 1 & 6 & 2 & 4 & 6 & 8 & 4 & 6 & 4 & 3 \\
\hline 7 & 2 & 2 & 4 & 6 & 2 & 7 & 4 & 6 & 6 & 12 \\
\hline 10 & 1 & 1 & 2 & 10 & 1 & 8 & 4 & 3 & 10 & 12 \\
\hline 11 & 2 & 1 & 4 & 4 & 2 & 3 & 4 & 3 & 4 & 22 \\
\hline 13 & 1 & 1 & 1 & 2 & 1 & 8 & 2 & 1 & 2 & 4 \\
\hline 14 & 1 & 4 & 2 & 4 & 4 & 7 & 2 & 12 & 4 & 10 \\
\hline 15 & 2 & 3 & 4 & 10 & 6 & 6 & 4 & 3 & 10 & 10 \\
\hline 17 & 1 & 4 & 1 & 6 & 4 & 8 & 1 & 12 & 6 & 4 \\
\hline 19 & 2 & 2 & 4 & 4 & 2 & 8 & 4 & 6 & 4 & 3 \\
\hline 21 & 1 & 1 & 2 & 4 & 1 & 14 & 2 & 3 & 4 & 4 \\
\hline 22 & 1 & 2 & 2 & 3 & 2 & 1 & 4 & 6 & 3 & 22 \\
\hline 23 & 2 & 4 & 4 & 2 & 4 & 3 & 4 & 12 & 2 & 10 \\
\hline 26 & 1 & 4 & 2 & 1 & 4 & 8 & 4 & 12 & 1 & 5 \\
\hline 29 & 1 & 4 & 1 & 1 & 4 & 1 & 2 & 4 & 1 & 4 \\
\hline 30 & 1 & 6 & 2 & 5 & 6 & 6 & 4 & 18 & 5 & 4 \\
\hline
\end{tabular}

\section{Vedic Composition Table: 4}

$\mathbf{2} \leq \mathrm{m} \leq \mathbf{1 1}$ and $\mathbf{5 3} \leq \mathrm{N} \leq 77$

\begin{tabular}{|c|c|c|c|c|c|c|c|c|c|c|}
\hline $\mathbf{g}_{\mathbf{N}}(\mathbf{m})$ & $\mathbf{m}=2$ & 3 & 4 & 5 & 6 & 7 & 8 & 9 & 10 & 11 \\
\hline $\mathbf{N}=53$ & 1 & 4 & 1 & 2 & 4 & 1 & 2 & 4 & 2 & 5 \\
\hline 55 & 1 & 2 & 1 & 10 & 2 & 8 & 2 & 6 & 10 & 11 \\
\hline 57 & 1 & 3 & 2 & 1 & 3 & 6 & 2 & 9 & 1 & 12 \\
\hline 58 & 1 & 1 & 2 & 6 & 1 & 3 & 4 & 1 & 6 & 1 \\
\hline 59 & 2 & 2 & 4 & 4 & 2 & 8 & 4 & 6 & 4 & 10 \\
\hline 61 & 1 & 1 & 1 & 2 & 1 & 8 & 2 & 1 & 2 & 4 \\
\hline 62 & 1 & 4 & 2 & 6 & 4 & 4 & 2 & 4 & 6 & 12 \\
\hline 65 & 1 & 4 & 1 & 10 & 4 & 3 & 1 & 12 & 10 & 12 \\
\hline 66 & 1 & 6 & 1 & 4 & 6 & 8 & 1 & 18 & 4 & 22 \\
\hline 67 & 2 & 2 & 4 & 3 & 2 & 3 & 4 & 2 & 6 & 10 \\
\hline 69 & 1 & 2 & 2 & 4 & 2 & 8 & 2 & 2 & 4 & 5 \\
\hline 70 & 1 & 2 & 2 & 1 & 2 & 14 & 4 & 6 & 1 & 5 \\
\hline 71 & 2 & 4 & 4 & 4 & 4 & 1 & 4 & 12 & 4 & 10 \\
\hline 73 & 1 & 1 & 1 & 2 & 1 & 8 & 1 & 3 & 2 & 12 \\
\hline 74 & 1 & 4 & 2 & 2 & 4 & 3 & 4 & 4 & 2 & 12 \\
\hline 77 & 1 & 4 & 2 & 1 & 4 & 7 & 2 & 4 & 1 & 22 \\
\hline
\end{tabular}


Vedic Composition Table: 5

$\mathbf{2 6} \leq \mathrm{m} \leq \mathbf{3 5}$ and $\mathbf{2} \leq \mathrm{N} \leq \mathbf{3 0}$

\begin{tabular}{|c|c|c|c|c|c|c|c|c|c|c|}
\hline $\mathbf{g}_{\mathbf{N}}(\mathbf{m})$ & $\mathbf{m}=26$ & 27 & 28 & 29 & 30 & 31 & 32 & 33 & 34 & 35 \\
\hline $\mathbf{N}=2$ & 14 & 36 & 6 & 10 & 12 & 15 & 16 & 12 & 8 & 6 \\
\hline 3 & 12 & 54 & 8 & 15 & 6 & 32 & 16 & 30 & 18 & 24 \\
\hline 5 & 14 & 12 & 8 & 7 & 20 & 5 & 8 & 20 & 6 & 40 \\
\hline 6 & 7 & 18 & 8 & 28 & 12 & 32 & 16 & 6 & 18 & 8 \\
\hline 7 & 14 & 18 & 28 & 28 & 6 & 15 & 4 & 12 & 6 & 42 \\
\hline 10 & 3 & 9 & 8 & 30 & 10 & 15 & 16 & 12 & 18 & 40 \\
\hline 11 & 14 & 9 & 12 & 15 & 4 & 32 & 16 & 22 & 18 & 12 \\
\hline 13 & 26 & 3 & 8 & 14 & 2 & 32 & 8 & 4 & 8 & 8 \\
\hline 14 & 12 & 36 & 14 & 6 & 4 & 3 & 8 & 20 & 9 & 28 \\
\hline 15 & 14 & 9 & 12 & 30 & 30 & 8 & 8 & 30 & 16 & 30 \\
\hline 17 & 6 & 36 & 8 & 30 & 12 & 32 & 4 & 4 & 34 & 24 \\
\hline 19 & 2 & 18 & 8 & 15 & 4 & 3 & 16 & 6 & 4 & 8 \\
\hline 21 & 14 & 9 & 14 & 5 & 4 & 16 & 8 & 4 & 16 & 28 \\
\hline 22 & 12 & 18 & 2 & 28 & 6 & 32 & 16 & 22 & 18 & 3 \\
\hline 23 & 12 & 36 & 12 & 28 & 4 & 16 & 4 & 20 & 18 & 6 \\
\hline 26 & 26 & 36 & 8 & 30 & 4 & 32 & 16 & 20 & 4 & 8 \\
\hline 29 & 2 & 4 & 1 & 58 & 4 & 32 & 8 & 4 & 6 & 1 \\
\hline 30 & 12 & 54 & 6 & 14 & 30 & 32 & 16 & 12 & 16 & 30 \\
\hline
\end{tabular}

Vedic Composition Table: 6

$26 \leq \mathrm{m} \leq \mathbf{3 5}$ and $\mathbf{5 1}<\mathrm{N}<\mathbf{8 0}$

\begin{tabular}{|c|c|c|c|c|c|c|c|c|c|c|}
\hline $\mathbf{g}_{\mathbf{N}}(\mathbf{m})$ & $\mathbf{m}=26$ & 27 & 28 & 29 & 30 & 31 & 32 & 33 & 34 & 35 \\
\hline $\mathbf{N}=53$ & 1 & 12 & 1 & 14 & 4 & 32 & 8 & 20 & 4 & 2 \\
\hline 55 & 12 & 18 & 8 & 15 & 10 & 8 & 8 & 22 & 16 & 40 \\
\hline 57 & 14 & 27 & 6 & 28 & 3 & 8 & 8 & 12 & 9 & 6 \\
\hline 58 & 2 & 3 & 6 & 58 & 6 & 32 & 16 & 1 & 18 & 6 \\
\hline 59 & 14 & 18 & 8 & 28 & 4 & 15 & 16 & 10 & 8 & 8 \\
\hline 61 & 1 & 3 & 8 & 10 & 2 & 32 & 8 & 4 & 6 & 8 \\
\hline 62 & 12 & 12 & 4 & 28 & 12 & 31 & 4 & 12 & 9 & 12 \\
\hline 65 & 26 & 36 & 3 & 14 & 20 & 32 & 2 & 12 & 18 & 30 \\
\hline 66 & 4 & 54 & 8 & 15 & 12 & 15 & 4 & 66 & 8 & 8 \\
\hline 67 & 2 & 2 & 12 & 28 & 6 & 15 & 16 & 10 & 2 & 3 \\
\hline 69 & 1 & 6 & 8 & 10 & 4 & 10 & 4 & 10 & 16 & 8 \\
\hline 70 & 7 & 18 & 14 & 30 & 2 & 15 & 16 & 10 & 16 & 14 \\
\hline 71 & 14 & 36 & 4 & 4 & 4 & 15 & 4 & 20 & 18 & 4 \\
\hline 73 & 14 & 9 & 8 & 30 & 2 & 32 & 4 & 12 & 18 & 8 \\
\hline 74 & 6 & 4 & 6 & 7 & 4 & 32 & 16 & 12 & 18 & 6 \\
\hline 77 & 4 & 4 & 14 & 10 & 4 & 32 & 4 & 44 & 16 & 7 \\
\hline 78 & 13 & 18 & 6 & 28 & 6 & 10 & 16 & 10 & 18 & 6 \\
\hline 79 & 12 & 6 & 12 & 30 & 4 & 16 & 4 & 12 & 18 & 12 \\
\hline
\end{tabular}


Vedic Composition Table: 7

$\mathbf{5 1} \leq \mathrm{m} \leq \mathbf{6 0}$ and $2 \leq \mathrm{N} \leq \mathbf{3 0}$

\begin{tabular}{|c|c|c|c|c|c|c|c|c|c|c|}
\hline $\mathbf{g}_{\mathbf{N}}(\mathbf{m})$ & $\mathbf{m}=51$ & 52 & 53 & 54 & 55 & 56 & 57 & 58 & 59 & 60 \\
\hline $\mathbf{N}=2$ & 8 & 14 & 54 & 36 & 12 & 12 & 20 & 10 & 20 & 12 \\
\hline 3 & 18 & 12 & 9 & 54 & 30 & 8 & 30 & 30 & 58 & 12 \\
\hline 5 & 12 & 14 & 18 & 12 & 10 & 8 & 12 & 7 & 29 & 20 \\
\hline 6 & 18 & 14 & 52 & 18 & 12 & 8 & 18 & 28 & 30 & 12 \\
\hline 7 & 6 & 28 & 52 & 18 & 12 & 28 & 18 & 28 & 58 & 12 \\
\hline 10 & 18 & 6 & 13 & 9 & 60 & 8 & 4 & 30 & 20 & 10 \\
\hline 11 & 18 & 28 & 52 & 18 & 44 & 12 & 6 & 30 & 30 & 4 \\
\hline 13 & 8 & 26 & 13 & 3 & 4 & 8 & 20 & 14 & 4 & 2 \\
\hline 14 & 36 & 12 & 54 & 36 & 20 & 14 & 20 & 6 & 60 & 4 \\
\hline 15 & 48 & 28 & 13 & 18 & 10 & 12 & 60 & 30 & 58 & 60 \\
\hline 17 & 68 & 6 & 13 & 36 & 12 & 8 & 36 & 30 & 29 & 12 \\
\hline 19 & 4 & 4 & 27 & 18 & 12 & 8 & 38 & 30 & 58 & 4 \\
\hline 21 & 16 & 14 & 9 & 9 & 4 & 14 & 10 & 5 & 29 & 4 \\
\hline 22 & 18 & 12 & 27 & 18 & 66 & 4 & 10 & 28 & 58 & 6 \\
\hline 23 & 36 & 12 & 54 & 36 & 10 & 12 & 36 & 28 & 12 & 4 \\
\hline 26 & 4 & 26 & 18 & 36 & 5 & 8 & 36 & 30 & 29 & 4 \\
\hline 29 & 12 & 2 & 26 & 4 & 4 & 2 & 20 & 58 & 29 & 4 \\
\hline 30 & 48 & 12 & 27 & 54 & 20 & 12 & 18 & 14 & 60 & 30 \\
\hline
\end{tabular}

Vedic Composition Table: 8

$51 \leq \mathrm{m} \leq 60$ and $51<\mathrm{N}<80$

\begin{tabular}{|c|c|c|c|c|c|c|c|c|c|c|}
\hline $\mathbf{g}_{\mathbf{N}}(\mathbf{m})$ & $\mathbf{m}=51$ & 52 & 53 & 54 & 55 & 56 & 57 & 58 & 59 & 60 \\
\hline $\mathbf{N}=53$ & 4 & 1 & 106 & 12 & 10 & 2 & 20 & 14 & 29 & 4 \\
\hline 55 & 16 & 12 & 54 & 18 & 110 & 8 & 18 & 15 & 6 & 10 \\
\hline 57 & 9 & 14 & 52 & 27 & 12 & 6 & 114 & 28 & 29 & 6 \\
\hline 58 & 18 & 2 & 18 & 3 & 6 & 12 & 9 & 58 & 60 & 6 \\
\hline 59 & 8 & 28 & 4 & 18 & 20 & 8 & 10 & 28 & 118 & 4 \\
\hline 61 & 6 & 1 & 6 & 3 & 4 & 8 & 3 & 10 & 4 & 2 \\
\hline 62 & 36 & 12 & 52 & 12 & 12 & 4 & 36 & 28 & 58 & 12 \\
\hline 65 & 36 & 26 & 54 & 36 & 60 & 3 & 20 & 14 & 60 & 20 \\
\hline 66 & 24 & 4 & 52 & 54 & 44 & 8 & 18 & 15 & 58 & 12 \\
\hline 67 & 2 & 4 & 27 & 2 & 30 & 12 & 10 & 28 & 15 & 12 \\
\hline 69 & 16 & 2 & 52 & 6 & 20 & 8 & 20 & 10 & 5 & 4 \\
\hline 70 & 16 & 14 & 52 & 18 & 5 & 28 & 20 & 30 & 60 & 2 \\
\hline 71 & 36 & 58 & 54 & 36 & 20 & 4 & 20 & 4 & 2 & 4 \\
\hline 73 & 18 & 14 & 54 & 9 & 12 & 8 & 9 & 30 & 60 & 2 \\
\hline 74 & 36 & 6 & 54 & 4 & 12 & 12 & 36 & 7 & 29 & 4 \\
\hline 77 & 16 & 4 & 13 & 4 & 22 & 14 & 12 & 10 & 20 & 4 \\
\hline 78 & 18 & 26 & 4 & 18 & 30 & 12 & 20 & 28 & 29 & 6 \\
\hline 79 & 18 & 12 & 6 & 6 & 12 & 12 & 20 & 30 & 58 & 4 \\
\hline
\end{tabular}




\section{CONCLUDING REMARKS}

* Brahmagupta's Bhāvanā has a logical concept in Ancient Indian mathematics. His logical system was responsible for the development of the Modern algebra, particularly in its implementation in Computer programming.

* BB-equation played important role in the evolution of Classical Algebra, Number theory and Computer Programming.

* Implicitly involves The principal of composition, a very important basic tool in Computer programming and Modern Algebra.

\section{ACKNOWLEDGEMENTS}

I acknowledge with gratitude and humanity my indebtedness to Dr. S.L.Singh, retired Professor and UGC emeritus fellowship,Gurukula Kangri University Haridwar, under whose guidance and support I had the privilege to complete this work.

\section{REFERENCES}

[1] Dutta B. and Singh. A.N, History of Hindu Mathematics, Part II, Asia publishing House (1962);

[2] Emch G.G, Sridharan. R and Srinivas. M.D, Contributions to the History of Indian Mathematics, CHOM 3, H.B.A (2005); p77-144.
[3] Nivan .Ivan, Zuckerman. S and Montgomery. L, An introduction to the Theory of Numbers, John Wiley (2000) p 47-128.

[4] Gallian J.A,Contemporary Abstract Algebra ,2 $2^{\text {nd }}$ Ed. Lexington, Mass: D.C.Heath,(1990).

[5] Fraleigh J.B, A first course in Abstract Algebra Pearson Education (2004) p40-230.

[6] Murthy T.S Bhanu, A modern introduction to ancient Indian mathematics New Delhi: Wiley Eastern Ltd; 1994.

[7] Swamy M.N.S, Brahmagupta's Theorem and recurrence relations A.M.S Classification Numbers.11B39, 33C25; (1998).

[8] Aggrawal V.S, Vedic Mathematics Delhi: Motilal Banarasidas.(1971);

[9] Weil Andre, Number Theory: An approach through history from Hamurapi to Legendre, Birkhuser (1984).

[10] Abell, Martha and Braselton, James.Equation with Maple, Academic press, 1999.

[11] Seshadri C.S, Studies in the History of Indian Mathematics,CHOM 5,H.B.A (2010); p192. 